\title{
BMJ Open The ongoing French metastatic breast cancer (MBC) cohort: the example-based methodology of the Epidemiological Strategy and Medical Economics (ESME)
}

David Pérol, ${ }^{1}$ Mathieu Robain, ${ }^{2}$ Patrick Arveux, ${ }^{3}$ Simone Mathoulin-Pélissier, ${ }^{4}$ Emmanuel Chamorey, ${ }^{5}$ Bernard Asselain, ${ }^{6}$ Delphine Berchery, ${ }^{7}$ Sophie Gourgou, ${ }^{8}$ Mathias Breton, ${ }^{9}$ Stéphanie Delaine-Clisant, ${ }^{10}$ Muriel Mons, ${ }^{11}$ Véronique Diéras, ${ }^{12}$ Matthieu Carton, ${ }^{12}$ Anne-Valérie Guizard,${ }^{13}$ Lilian Laborde, ${ }^{14}$ Carine Laurent, ${ }^{15}$ Agnès Loeb, ${ }^{16}$ Marie-Ange Mouret-Reynier, ${ }^{17}$ Damien Parent, ${ }^{18}$ Geneviève Perrocheau, ${ }^{19}$ Loïc Campion, ${ }^{19}$ Michel Velten, ${ }^{20}$ Christian Cailliot, ${ }^{2}$ Monia Ezzalfani, ${ }^{1}$ Gaëtane Simon ${ }^{2}$

To cite: Pérol D, Robain M, Arveux $\mathrm{P}$, et al. The ongoing French metastatic breast cancer (MBC) cohort: the example-based methodology of the Epidemiological Strategy and Medical Economics (ESME). BMJ Open 2019;9:e023568. doi:10.1136/ bmjopen-2018-023568

- Prepublication history for this paper is available online. To view these files, please visit the journal online (http://dx.doi. org/10.1136/bmjopen-2018023568).

Received 12 April 2018 Revised 19 November 2018 Accepted 3 December 2018

Check for updates

(C) Author(s) (or their employer(s)) 2019. Re-use permitted under CC BY-NC. No commercial re-use. See rights and permissions. Published by BMJ.

For numbered affiliations see end of article.

Correspondence to

Dr David Pérol;

david.perol@lyon.unicancer.fr

\section{ABSTRACT}

Purpose The currently ongoing Epidemiological Strategy and Medical Economics (ESME) research programme aims at centralising real-life data on oncology care for epidemiological research purposes. We draw on results from the metastatic breast cancer (MBC) cohort to illustrate the methodology used for data collection in the ESME research programme.

Participants All consecutive $\geq 18$ years patients with MBC treatment initiated between 2008 and 2014 in one of the 18 French Comprehensive Cancer Centres were selected. Diagnostic, therapeutic and follow-up data (demographics, primary tumour, metastatic disease, treatment patterns and vital status) were collected through the course of the disease. Data collection is updated annually.

Finding to date With a recruitment target of 30000 patients with MBC by 2019 , we currently screened a total of 45329 patients, and $>16700$ patients with a metastatic disease treatment initiated after 2008 have been selected. $20.7 \%$ of patients had an hormone receptor (HR)-negative $\mathrm{MBC}, 73.7 \%$ had a HER2-negative $\mathrm{MBC}$ and $13.9 \%$ were classified as triple-negative $\mathrm{BC}$ (ie, HER2 and HR status both negative). Median follow-up duration from MBC diagnosis was 48.55 months for the whole cohort.

Future plans These real-world data will help standardise the management of MBC and improve patient care. A dozen of ancillary research projects have been conducted and some of them are already accepted for publication or ready to be issued. The ESME research programme is expanding to ovarian cancer and advanced/metastatic lung cancer. Our ultimate goal is to achieve a continuous link to the data of the cohort to the French national Health Data System for centralising data on healthcare reimbursement (drugs, medical procedures), inpatient/outpatient stays and visits in primary/secondary care settings.

Trial registration number NCT03275311; Pre-results.

\section{Strengths and limitations of this study}

The Epidemiological Strategy and Medical Economics research programme aims at centralising real-life data on oncology care for epidemiological research purposes. The ongoing screening of the metastatic breast cancer (MBC) cohort reached $>16700$ patients with metastatic disease treatment initiated after 2008 , currently contributing to the development of one of the most important cohort of patients with treated MBC.

- Screening process of patients and data collection (diagnostic, therapeutic and follow-up data) through the course of the disease provide a solid base of knowledge for real-world survival. The significant resources deployed allowed to achieve a high quality-level data validation, including systematic consultancy of the source folder for data collection, and the implementation of effective quality control, and regular audit.

- The main limitations are (1) the lack of availability of electronic medical records data required to describe the global MBC management due to the low level of standardisation of current electronic medical records and (2) the retrospective patient selection-data collection, notwithstanding the prospective compilation of real-life follow-up, clinical and biological events, preventing to assess several endpoints classically defined for randomised clinical trials such as progression disease at predefined time points.

- Despite the current large-scale recruitment of patients and greater than one-third of French patients with MBC managed in French Comprehensive Cancer Centres, future studies should integrate the diversity of management options adopted in any health institutions. 


\section{BACKGROUND}

Real-world evidence (RWE) studies and observational studies using real-world data (RWD) play a growing role in conducting comparative effectiveness research on pharmaceutical products and other healthcare interventions. They aim to bridge the gap between the highly controlled environment of randomised clinical trials (RCTs) and real-life clinical practice. ${ }^{1}$ In particular, health authorities are interested in gathering RWD for long-term benefitrisk assessment and, increasingly, health economic evaluations for reimbursement decisions. ${ }^{2}$ With their high internal validity, RCTs are considered the gold standard of evidence for establishing treatment efficacy, although the generalisability of findings to clinical practice may be limited. ${ }^{3}$ In fact, cancer survival endpoints in the real-life setting may differ from that measured in traditional RCTs. ${ }^{4}$ Furthermore, other limitations such as short follow-up and small sample size have created uncertainty in estimating survival criteria in RCTs, and thus surrogate endpoints are generally used such as progression-free survival (PFS) or time to progression. ${ }^{5}$ On the other hand, large population-based cohorts with longer follow-up periods can be particularly appropriate to assess long-term clinical outcomes, such as overall survival (OS) outside of the RCT setting ${ }^{6}$ and to detect changes in medical practice.

RWE and observational studies are non-experimental research where cancer management (treatments and disease evolution assessment) is left to the choice of care providers and patients. ${ }^{7}$ Over the past few years, a broad consensus arose around the requirement for high-quality data from large cohorts to strengthen and drive improvements in research methods and practices. ${ }^{8}$ The goal of these studies is to generate complementary information to RCTs based on larger samples and provide answers regarding particular populations in real-life clinical practice. These studies are more prone to biases such as baseline differences between patients (selection bias) or bias due to confounding by indication for example. To minimise sources of such biases, statistical approaches including adjusted analyses, propensity score methods or instrumental variables may be also employed. ${ }^{9-12}$

Metastatic breast cancer (MBC) is one of the leading causes of cancer-related mortality among women in Western countries. ${ }^{13}$ A relatively high proportion (approximately $30 \%$ ) of patients with breast cancer develop metastatic disease, ${ }^{14}$ and while significant treatment advances have been made, the overall prognosis is poor with a 5-year survival rate of $25 \% .^{15}$ The national academic network of cancer centres in France (French Comprehensive Cancer Centres (FCCCs)), which together handle over one-third of all breast cancer cases nationally, decided to launch in 2014 a programme dedicated to focusing on RWD in oncology databases in MBC through Epidemiological Strategy and Medical Economics (ESME) research programme.

The ESME research programme aims to build a comprehensive database on oncology care for epidemiological research purposes to improve knowledge on medical practice in real-life setting, on public health and healthcare use, and to provide information to health authorities and other associated bodies.

Several studies based on real-life data collection have been developed in this programme. Different cohorts of patients with ovarian cancer and patients with advanced/ metastatic lung cancer are currently recruiting.

Some results from the ongoing ESME MBC project ( ClinicalTrials.gov NCT03275311) whose aim is to describe the medical care of patients treated for MBC according to their disease characteristics the evolution of their metastatic disease and their outcomes have been recently published. Recent analyses explored OS in different subgroups of patients with MBC, and first-line therapy in patients with HER2-negative $\mathrm{MBC}$, and in patients with hormone receptor-positive HER2-negative MBC. ${ }^{16-18}$

In this paper, we describe the methodological principles that underpin the ESME research programme, and illustrate this innovative approach through this first ESME MBC cohort. Design, brief description of the selected population and current status are reported.

\section{METHODS}

The ESME MBC cohort is a population-based registry in 18 FCCCs (http://www.unicancer.fr/en/rd-unicancer/ esme), which collected data on all consecutive patients treated for MBC from 2008. Annual data collection phases are planned to add new diagnosed cases and update patients' follow-up data.

\section{STUDY POPULATION \\ Eligibility criteria}

Patients eligible to the ESME MBC cohort were male or female patients aged $\geq 18$ years with $\mathrm{MBC}$ whose first metastasis had been either completely or partially treated between 1 January 2008 and 31 December 2014 in one of the FCCCs. MBC treatment could include radiotherapy, chemotherapy, targeted therapy, immunotherapy or endocrine therapy.

\section{Patient screening process}

Patient screening process involved two steps: an automated case screening followed by the validation of selection for each screened case. The automated case screening was based on information retrieved from multiple data sources available within each FCCC: administrative records (French National Computerised Medical Information System) (via MBC-specific International Classification of Diseases (ICD) codes associated with inpatient stays), pharmacy records, patient medical records (PMRs), including multidisciplinary team meeting records and search using relevant keywords, MBC-specific registries.

The objective of the first step-automated screening step-was to identify all cases with inpatient stays or therapeutic management for MBC in one of the FCCCs during 
the selection period and generate the patient screening list. The ICD codes used were C50 (Malignant neoplasm of breast), C77.- (except C77.3) (Secondary and unspecified malignant neoplasm of lymph nodes), C78.- (Secondary malignant neoplasm of respiratory and digestive organs) and C79.- (Secondary malignant neoplasm of other and unspecified sites). Once the patient screening list was finalised in each centre, data were subsequently anonymised and each patient had been assigned an ESME number. The first screening step did not allow to precisely identify the date for first MBC-specific treatment, and the second step was performed to cross-check eligibility criteria for all screened cases and specify the dates related to the initiation of the MBC first-line treatment, using data from the PMRs.

\section{Patient data protection}

The ESME research programme was managed by R\&D Unicancer in accordance with guidelines for Good Pharmacoepidemiology Practices and Good Epidemiology Practices. ${ }^{19} 20$

Ancillary projects analyses were notified to an independent ethics committee (Lyon Sud-Est II) on 17 December 2015 .

\section{Data collection}

The data of the selected patients were planned to be collected by trained research assistants and annually updated. The data collection was performed in two phases from October 2014 to October 2016. A first data collection phase conducted in 2014-2015 collected the data from patients with MBC treatment initiated

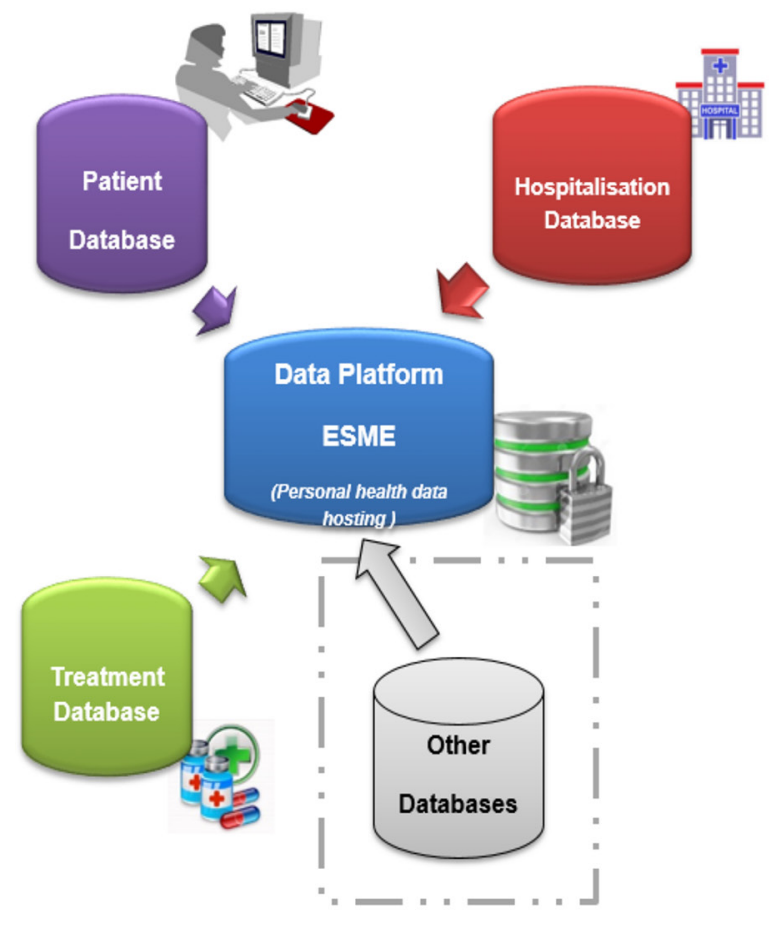

between 1 January 2008 and 31 December 2013 in one of the FCCCs. A second phase of data collection performed in 2016 added to the ongoing database the data from patients with MBC treatment initiated between 1 January and 31 December 2014, and follow-up data for the global cohort were consequently updated. Hence, the ongoing database provides an overview of all the data from patients with an MBC treatment initiated from 2008; information is updated with last contact information available in the PMR at the date of the data collection.

\section{Baseline and follow-up data}

The ESME MBC cohort is composed of three types of data set (figure 1):

Patient-related data are obtained from systematic review of patient medical records (non-structured data) and provide information on patient demographics, cancer family history, characteristics of primary tumour, relapses, metastatic recurrences pathological reports (tumour size, grade, histological type), hormone-receptor status and HER-2 status, therapeutic care (focusing on cancer-related treatment) and settings, reasons for treatment termination and clinical events.

Hospitalisation records are integrated data from a structured and automated database related to inpatient stays, and primarily used to bill the French National Health Insurance Fund (Assurance Maladie). It provides information on patient entry and discharge information (date and destination code at discharge), ICD codes associated to each stay, diagnostic, medical

\section{Hospitalisation Database}

- Hospitalisations: dates, diagnoses, GHS code

- Medical procedures (Inc. Radiotherapy): dates, code

\section{Treatment Database}

" Pharmacy records: dates, antineoplastic drugs, therapeutic protocol and other concomitant drugs

\section{Patient Database}

- Collection based on Electronic medical records

- Patient data : demographics, cancer management, clinical events (progression, relapse), pathological report, metastatic disease, anti-cancer treatment (chemotherapy, targeted therapy, endocrine therapy, immunotherapy), and other therapeutic care ( radiotherapy, surgery) or supportive care

Figure 1 The Epidemiological Strategy and Medical Economics (ESME) data platform. GHS, Hospital stay related group. 


\begin{tabular}{|c|c|c|}
\hline Patient-related & Hospitalisation-related & Pharmacy record-related \\
\hline Patient data & Age & Height \\
\hline Demographics & Gender & Body mass index \\
\hline Other cancer and family history & Main diagnosis code (ICD code) & Drug (International Non-proprietary Name) \\
\hline Menopausal status & Linked diagnosis code (ICD code) & Protocol \\
\hline Initial tumour: & Related significant diagnoses (ICD code) & Cycle ID \\
\hline Diagnosis & $\begin{array}{l}\text { Diagnosis-related group code } \\
\text { (reimbursement coding) }\end{array}$ & Pharmaceutical form and dosage \\
\hline Relapses & $\begin{array}{l}\text { Hospital stay-related group ID } \\
\text { (reimbursement coding) }\end{array}$ & Administration date \\
\hline Histological results & Entry date & Line treatment number \\
\hline Medical care & Discharge date & Administered dose \\
\hline Metastatic disease: & Destination code at discharge & Cumulated dose \\
\hline Diagnosis & Medical procedures performed & Inclusion in a clinical trial (yes/no) \\
\hline Progression & Radiation therapy & \\
\hline \multicolumn{3}{|l|}{ Histological results } \\
\hline \multicolumn{3}{|l|}{ Therapies } \\
\hline \multicolumn{3}{|l|}{$\begin{array}{l}\text { Invasive procedures related to } \\
\text { metastasis }\end{array}$} \\
\hline \multicolumn{3}{|l|}{ Last contact: } \\
\hline \multicolumn{3}{|l|}{ Vital status } \\
\hline Date of last contact/death & & \\
\hline
\end{tabular}

ICD, International Classification of Diseases.

and therapeutic procedures including radiotherapy and surgery.

Pharmacy-dispensed treatment records includes all data related to anticancer treatments obtained from each centre's pharmacy database: drugs (International Non-proprietary Name), administration date, protocol name, patient's height and body mass index, cycle ID, common unit of delivery (UCD) code (pharmaceutical form related to the drug dosage), line of treatment and administration in a clinical trial (yes/no). It exclusively includes information on products that are prescribed by each FCCC.

The detailed raw data collected above and derived data are listed in table 1.

\section{DATA MANAGEMENT}

Any data integrated in the ESME research programme are subject to quality control (QC) procedures.

Patient-related data are registered via an electronic case report form (eCRF) in each centre by trained clinical research assistants (CRAs) between December 2014 and October 2016. Medical support to assist CRAs was provided to ensure QC data and appropriate recoding was performed before annual database lock when required. All ESME procedures are handled according to the Guidelines for Good Pharmacoepidemiology Practices. ${ }^{20}$ Importantly, all data are exclusively obtained retrospectively; no attempts are made to recover unavailable data from PMRs by contacting healthcare providers or patients.

The clinical data management system used was SAS software V.9.4. For both, ESME MBC database and the eCRF tool administration used an Oracle solution and certified personal data hosting system guarantee data security.

\section{On-site quality review}

On-site quality review of the patient screening process was carried out. This consisted of checking eligibility criteria on samples of selected and non-selected cases in each FCCC. For selected cases, key variables were crosschecked versus the source data. For all QC procedures, accepted error limits were $10 \%$ for non-selected patients and $5 \%$ for selected patients. A central audit was subsequently performed by the Unicancer Quality Assurance Department, and an audit on data registration and generated screening list was also conducted at the local level.

\section{DATA QUALITY ASSURANCE \\ Governance structure}

Three boards monitor the ESME Research programme: Scientific Committee, Deontology Committee and International Advisory Board. The main role of the Scientific Committee is to (1) ensure that the applicable scientific rules are followed, (2) evaluate any ancillary projects 


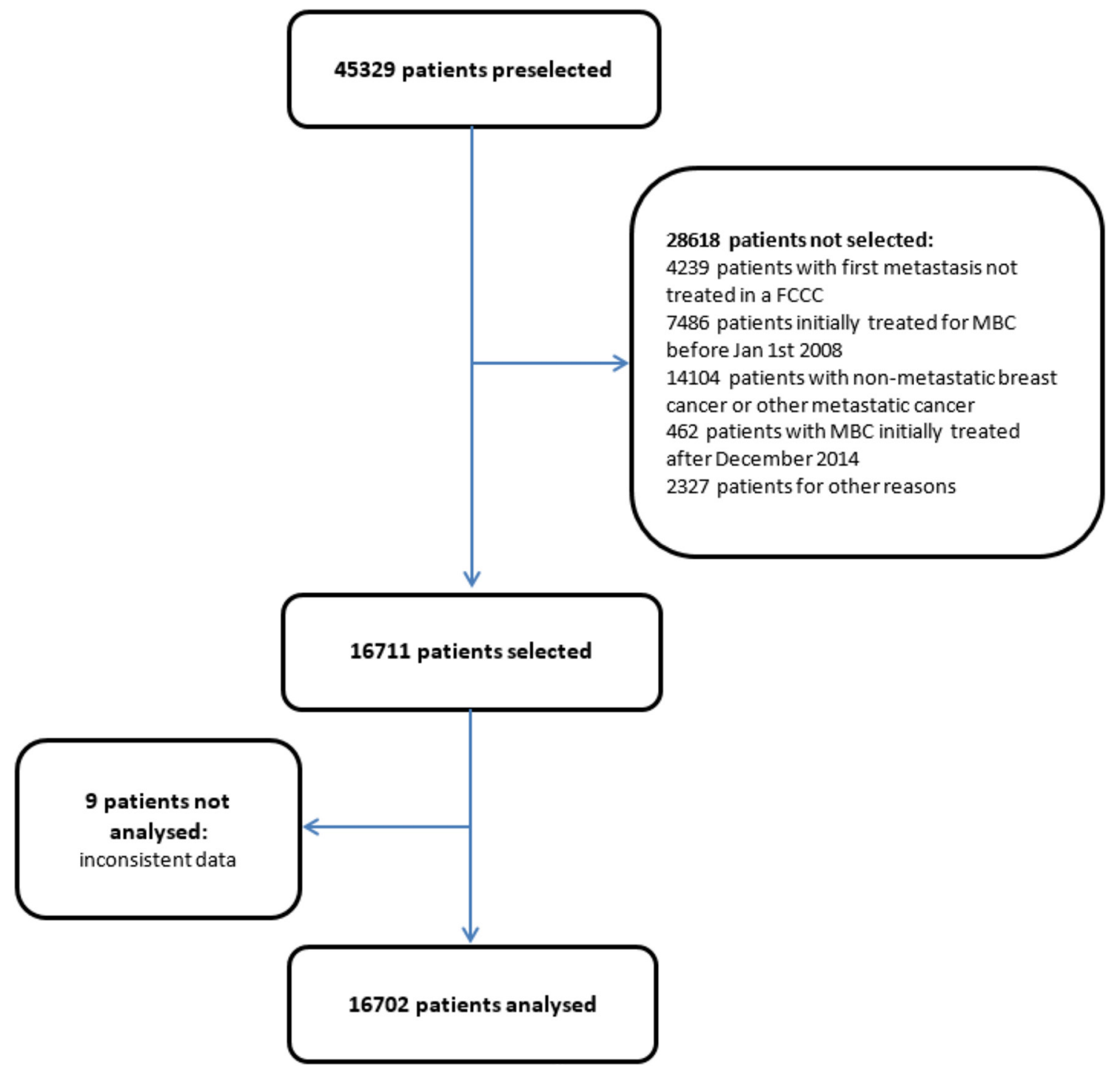

Figure 2 Epidemiological Strategy and Medical Economics metastatic breast cancer (MBC) cohort: flow chart. FCCC, French Comprehensive Cancer Centre.

in compliance with defined criteria and scientific pertinence, and (3) monitor all the validated ancillary projects. The Deontology Committee monitors any potential conflicts of interest related to experts involved in the programme, gives recommendations that may improve the prevention of conflicts, provides opinions on individual or particular situations, and potential collaborations with private partners. The ESME International Advisory Board has a consultative role with regard to coherence of the scientific programme and reviews key international communications, formulates recommendations for publication rules or methodology and reinforces international academic cooperation.

\section{DATA ANALYSIS PRINCIPLES}

Academic research teams or private organisations could propose ancillary projects.

For each accepted ancillary project, statistical analyses are conducted according to a detailed statistical analysis plan that must be reviewed by the scientific committee. This article does not aim at providing a comprehensive and exhaustive review on appropriate statistical methods to reduce bias related to analysis based on RWD.

A first ancillary project reported the outcomes (OS and PFS) following first-line paclitaxel treatment with or without bevacizumab. ${ }^{16}$ Other ongoing analyses of sensibility will better address the bias potentially found in real-life settings. Two analyses reported the description of OS in different subgroups of patients with MBC over the time, and results for the first-line therapy (endocrine therapy or chemotherapy) in hormone receptor-positive HER2 negative cancer subgroup, respectively. ${ }^{17} 18$ Other series accepted for communications (abstracts/posters) to major congress in 2017 reported epidemiological analyses (ie, impact of age at diagnosis, etc), therapeutic management (ie, impact of loco-regional treatment on OS, etc) and specific analyses for drug use in routine practice (ie, use of vinorelbine, everolimus, etoposide, etc). 
Different other subpopulations are currently being considered such as subgroups of metastatic triple-negative breast cancer, metastatic HER2-positive MBC, etc.

\section{PATIENT AND PUBLIC INVOLVEMENT STATEMENT}

Patients and/or public were not involved.

\section{RESULTS AND CURRENT STATUS}

In total, 16711 out of the 45329 patients screened were selected in the ESME MBC cohort (see figure 2).

The sensitivity and specificity of the three main screening sources used were explored. Sensitivity was highest for administrative records $(78 \%$ vs $43 \%$ for pharmacy records, and 28\% for BC-specific local registries). On the other hand, specificity was highest for BC-specific local registries $(87 \%$ vs $67 \%$ for pharmacy records, and $49 \%$ for administrative records).

The main reasons for non-selection of screened patients were presence of non-MBC or other metastatic cancers ( $\mathrm{n}=14104$ patients), initial MBC treatment received before 1January $2008(\mathrm{n}=7486)$, and first metastasis not initially treated in an FCCC $(n=4239)$. Nine additional were excluded prior to the final database lock due to inconsistencies in the dates. A total of 16702 patients were analysed (figure 2).

Table 2 summarises the main demographic and disease characteristics at the time of initial metastatic diagnosis. Patients were nearly all women $(99.1 \%)$ with a median age of 61 years. Over half $(56.2 \%)$ had at least visceral metastases present, with $30.2 \%$ having at least bone and non-visceral metastases, and $13.6 \%$ with skin only, or node only, or at least skin plus node. $20.7 \%$ of patients had an HR-negative MBC, 73.7\% had a HER2-negative MBC and $13.9 \%$ were classified as triple-negative BC (ie, HER2 and HR status both negative).

Median follow-up duration from MBC initiation treatment was 48.55 months for the whole cohort $(95 \%$ CI 47.7 to 49.38$)$.

\section{DISCUSSION}

Retrospective analysis using RWD is likely to become increasingly important to ensure that medications are accepted by policymakers and adopted by patient practitioners. The ESME Research programme is a large-scale initiative to provide access to RWD in oncology. This ongoing ESME MBC cohort currently centralises data from 16711 patients.

The ESME research programme provides a unique opportunity to study a diverse range of topics related to MBC care and management in real-life settings. Indeed, there are many potential applications, including study of the factors influencing patient care (eg, cancer and patient characteristics), description of therapeutic strategies (treatment lines and sequences of therapies, etc) and measurement of clinical events (disease progression,
Table 2 Characteristics of the patients at of the date of metastatic disease diagnosis

\begin{tabular}{|c|c|}
\hline Characteristic & ESME MBC population $(n=16$ 702) \\
\hline \multicolumn{2}{|l|}{ Age (years) } \\
\hline Mean (SD) & $60.6(13.8)$ \\
\hline Median (Q1-Q3 range) & $61.0(51.0-71.0)$ \\
\hline \multicolumn{2}{|l|}{ Sex } \\
\hline Male & $149(0.9 \%)$ \\
\hline Female & $16553(99.1 \%)$ \\
\hline \multicolumn{2}{|c|}{ Histological grade* at primary tumour diagnosis } \\
\hline 1 & $1277(10.1 \%)$ \\
\hline 2 & $6438(50.7 \%)$ \\
\hline 3 & $4733(37.3 \%)$ \\
\hline \multicolumn{2}{|l|}{ Metastatic status $\dagger$} \\
\hline Not available & $240(1.9 \%)$ \\
\hline Missing data & 2008 \\
\hline De novo MBC & $4763(28.5 \%)$ \\
\hline Relapsed MBC & $11939(71.5 \%)$ \\
\hline \multicolumn{2}{|c|}{ Year of first metastatic treatment } \\
\hline 2008 & $2651(15.9 \%)$ \\
\hline 2009 & $2675(16.0 \%)$ \\
\hline 2010 & $2598(15.6 \%)$ \\
\hline 2011 & $2515(15.1 \%)$ \\
\hline 2012 & $2371(14.2 \%)$ \\
\hline 2013 & $2216(13.3 \%)$ \\
\hline 2014 & $1676(10.0 \%)$ \\
\hline \multicolumn{2}{|l|}{ Type of metastases } \\
\hline Visceral & $9383(56.2 \%)$ \\
\hline Bones and not visceral & 5047 (30.2\%) \\
\hline Nodes only & $880(5.3 \%)$ \\
\hline Skin only & $916(5.5 \%)$ \\
\hline Skin+nodes & $476(2.8 \%)$ \\
\hline \multicolumn{2}{|l|}{ Global HR status $\ddagger$} \\
\hline Positive & $12748(76.3 \%)$ \\
\hline Negative & $3451(20.7 \%)$ \\
\hline Not determined & $503(3.0 \%)$ \\
\hline \multicolumn{2}{|l|}{ Global HER2 statusł } \\
\hline Positive & $2863(17.1 \%)$ \\
\hline Negative & 12306 (73.7\%) \\
\hline Not determined & $1533(9.2 \%)$ \\
\hline \multicolumn{2}{|l|}{ Triple-negative status } \\
\hline Yes & $2321(13.9 \%)$ \\
\hline
\end{tabular}

Data are $\mathrm{n}(\%)$ unless indicated otherwise.

${ }^{*}$ Histological grade at primary tumour diagnosis: the histological grade at primary tumour diagnosis is defined as the worst histological grade recorded within 1 month (30 days) after the initial diagnosis (primary tumour). †MBC is considered de novo if the diagnosis of metastatic disease occurs within 6 months (180 days) after the initial diagnosis (primary tumour). †The ER or PR is considered positive if the pathology report indicates a 'positive' result or considered as positive when $\geq 10 \%$ of cells in the sample are positive for ER or PR, respectively. The HER2 status is considered positive if the pathology report indicates for the immunohistochemistry result ' $3+$ ', ' $2+$ ' or not available, the result will be considered positive if the fluorescent in situ hybridisation or chromogenic in situ hybridisation result is positive. If two or more histological reports are available at the same date, the positive status is preferred. The global HR/HER2 status indicates the status at metastatic diagnosis based on histological results forms related to the primary tumour (if available) or metastatic sites.

ER, oestrogen receptor; ESME, Epidemiological Strategy and Medical Economics; MBC, metastatic breast cancer; PR, progesterone receptor status. 
death, persistence of treatment effect). Therefore, this approach allows a better characterisation of patients enroled in clinical trials and contributes to simulation of trials using appropriate statistical methodologies. Potentially, these data could be used for health economics evaluation of management strategies for patients (eg, rehospitalisation and related ambulatory care), as well as reconstruction of healthcare trajectories through data modelling.

The ESME research programme includes alternative approaches to generate cohorts that use different types of RWD (clinical data, therapeutic treatment data, longterm outcomes, health economics data) in the FCCCs versus existing registries in France, Europe and the USA (eg, SEER). It involves rigorous procedures for patient screening and data collection, ensuring both validity and reliability of data. It uses a fully retrospective approach, with no influence on treatment practice or interaction with oncologists. Unlike prospective interventional or observational research studies, data are not influenced by study design and reflect the real-life management of patients treated. While data recorded for the cohort are defined by experts in the field, the vast majority of data are collected by trained clinical research technicians, thereby minimising any potential risk of data misinterpretation. As discussed above, the ESME MBC cohort offers a unique opportunity to study a wide range of research questions in a large sample. With respect to evaluation of treatment strategies, the database enables reliable estimation of survival criteria such as OS and surrogates endpoints (PFS, etc). OS improvement in diseases with a long median post-progression survival time, such as MBC, is a critical endpoint. ${ }^{21-23}$

The ESME MBC cohort also has several limitations. For example, the database relies on the collection and restructuring of existing data only, that is, there is no creation of new data. Furthermore, apart from events reported in the PMR impacting therapeutic management, adverse effects are not routinely captured. Conceivably, further in-depth analysis of the data could highlight trends such as treatment interruption or discontinuation due to toxicity, which is important from a risk management perspective. The main potential sources of bias include selection bias, and information bias due to differences in patient monitoring and non-standardised data collection. Selection bias has been taken into account by using rigorous selection procedures across all 18 FCCCs, and the data management plan and QC programme described above have been designed to limit information bias. Nevertheless, due to the retrospective data collection and the fact that it is based on real-life follow-up, clinical and biological events are not evaluated at predefined time points (unlike in RCTs). For example, objective response, historical endpoint in RCTs, could not be assessed retrospectively without a central review of existing imaging as not systematically documented in routine practice. The information collected therefore depends on the frequency of follow-up visits and clinical and radiological exams prescribed by the patient's doctor. As clinical signs are the only means by which disease metastasis can be identified, the number of disease progressions may be underestimated. With respect to the clinical event of death, all deaths are reported in the PMRs.

With respect to evaluation of treatment strategies, analysis of real-life data poses unique challenges, such as accounting for confounding factors between patient groups, although various statistical approaches can be used to address this, as discussed above. ${ }^{24}$

Concerning overall generalisability and applicability (external validity), it should be noted that the cohort centralises data from patients treated in specialised cancer centres only. FCCCs may use different clinical practices compared with public hospitals and private institutions, and thus patients from FCCCs may not be truly representative of all French patients with breast cancer. Potentially, data extrapolation from all French healthcare organisations could be developed with the Exhaustive National Health Reimbursement System (Système national d'information inter-régimes de l'Assurance maladie).

The ESME MBC cohort aims to collect data for up to 30000 patients by 2019. As mentioned, future aims might include to continuously link our database to those from other institutions, such as the SNDS database for data on exhaustive healthcare reimbursement, and the INSEE database to provide vital status updates for patients lost to follow-up. The ESME research programme has further expanded to ovarian cancer and advanced/metastatic lung cancer. RWD from the ESME cohorts should help to provide medical recommendations and ultimately improve patient care. ${ }^{25}$

\section{Author affiliations}

${ }^{1}$ Biostatistic Unit, Clinical Research and Innovation Department, Centre Léon Bérard, Lyon, France

${ }^{2}$ R\&D UNICANCER, Paris, France

${ }^{3}$ Registre des cancers du sein de Côte d'Or, centre Georges-François-Leclerc, Dijon, France

${ }^{4}$ Clinical and Epidemiological Research Unit, INSERM CIC1401, Institut Bergonie, Comprehensive Cancer Center, Bordeaux, France

${ }^{5}$ Centre Antoine-Lacassagne, Nice, France

${ }^{6}$ University Paris Sud, Paris, France

${ }^{7}$ Institut Claudius Regaud, Toulouse, France

${ }^{8}$ Institut régional du Cancer Montpellier, Montpellier, France

${ }^{9}$ Centre Eugene Marquis, Rennes, France

${ }^{10}$ Clinical Research Unit, Centre Oscar Lambret, Lille, France

${ }^{11}$ Gustave Roussy, Villejuif, France

${ }^{12}$ Ensemble Hospitalier de I'Institut Curie, Paris \& Saint-Cloud, France

${ }^{13}$ Centre François Baclesse, Caen, France

${ }^{14}$ Institut Paoli-Calmettes, Marseille, France

${ }^{15}$ Institut de Cancerologie de Lorraine, Vandoeuvre-les-Nancy, France

${ }^{16}$ Centre Henri Becquerel, Rouen, France

${ }^{17}$ Centre Jean Perrin, Clermont-Ferrand, France

${ }^{18}$ Institut Jean Godinot, Reims, France

${ }^{19}$ Institut de Cancérologie de l'Ouest, Nantes \& Angers, France

${ }^{20}$ Centre Paul Strauss, Strasbourg, France

Acknowledgements The authors thank the 18 French Comprehensive Cancer Centres for providing the data and each ESME contact for coordinating the project at the local level. We thank the ESME Scientific Committee members for their ongoing support. They also acknowledge Dr Sarah Hopwood (Scinopsis, France) for editorial assistance with this publication, paid by R\&D UNICANCER. The 18 French 
Comprehensive Cancer Centres (FCCC) are Ensemble hospitalier de l'Institut Curie, Paris/Saint-Cloud, Gustave Roussy, Villejuif, Institut de Cancérologie de l'Ouest, Nantes/Angers, Centre François Baclesse, Caen, Institut du Cancer de Montpellier Montpellier, Centre Léon Bérard, Lyon, Centre Georges-François Leclerc, Dijon, Centre Henri Becquerel, Rouen; Institut Claudius Régaud, Toulouse; Centre Antoine Lacassagne, Nice; Institut de Cancérologie de Lorraine, Nancy; Centre Eugène Marquis, Rennes; Institut Paoli-Calmettes, Marseille; Centre Jean Perrin, ClermontFerrand; Institut Bergonié, Bordeaux; Centre Paul Strauss, Strasbourg; Institut Jean Godinot, Reims; Centre 0scar Lambret, Lille. ESME central coordinating staff: Head of Research and Development: Christian Cailliot. Program director: Mathieu Robain. Data Managers: Irwin Piot and Olivier Payen. Project Managers: Coralie Courtinard, Tahar Guesmia and Gaëtane Simon. Project assistant: Esméralda Pereira. Software designer: Alexandre Vanni. ESME local coordinating staff: Patrick Arveux, Thomas Bachelot, Jean-Pierre Bleuse, Delphine Berchery, Mathias Breton, Stéphanie Clisant, Emmanuel Chamorey, Valérie Dejean, Véronique Diéras, Anne-Valérie Guizard, Anne Jaffré, Lilian Laborde, Agnès Loeb, Muriel Mons, Damien Parent, Geneviève Perrocheau, Marie-Ange Mouret-Reynier, Carine Laurent, Michel Velten.

Contributors DP and MR: conception and design, writing the first draft. PA, DB, A-VG and M-AM-R: conception and design, data acquisition. SM-P: conception and design, interpretation. EC and LC: data acquisition and statistical analysis. BA, SG and MC: statistical analysis. MB, SC, MM, VD, LL, CL, AL, DP and GP: data acquisition. MV: conception and design, data acquisition and statistical analysis. CC: conception and design. ME: statistical analysis and writing the first draft. GS: conception and design, statistical analysis and writing the first draft. All authors reviewed and approved the final version of the manuscript.

Funding This work was supported by R\&D UNICANCER and initially funded by an industrial consortium (Roche, Pierre Fabre, Pfizer, and Astrazeneca), and support was subsequently provided by MSD, Eisai,and Daiichi Sankyo.

Disclaimer The funders did not have any input into maintenance of the database (project design, data collection, management) or analysis of data. They were not involved in the writing of the manuscript or decision to submit for publication.

Competing interests DP has received personal fees (honoraria and travel/ accommodation expenses) from Laboratoire Roche, outside the submitted work. BA has received personal fees for board membership and for consultancy from Roche Pharma, outside the submitted work. SG has received personal fees for board membership from Celgene and for consultancy from Roche, outside the submitted work.

\section{Patient consent Not required.}

Ethics approval The ESME MBC database received approval from the French data protection authority (Commission Nationale de l'Informatique et des Libertés, authorisation no. 1704113).

Provenance and peer review Not commissioned; externally peer reviewed.

Data sharing statement We reported the methodology developed to collect and control the data of the large ESME programme and illustrated the methodology with data from the cohort of patients with metastatic breast cancer patients. Data collected are listed in table 1. The database of the ESME programme or the database of the MBC cohorts are currently not accessible. For any specific demand, please contact the corresponding author. Each demand will be examined on a caseby-case basis by the scientific committee.

Open access This is an open access article distributed in accordance with the Creative Commons Attribution Non Commercial (CC BY-NC 4.0) license, which permits others to distribute, remix, adapt, build upon this work non-commercially, and license their derivative works on different terms, provided the original work is properly cited, appropriate credit is given, any changes made indicated, and the use is non-commercial. See: http://creativecommons.org/licenses/by-nc/4.0/.

\section{REFERENCES}

1. Chavez-MacGregor M, Giordano SH. Randomized clinical trials and observational studies: is there a battle? J Clin Oncol 2016;34:772-3.

2. Berger ML, Mamdani M, Atkins D, et al. Good research practices for comparative effectiveness research: defining, reporting and interpreting nonrandomized studies of treatment effects using secondary data sources: the ISPOR Good Research Practices for Retrospective Database Analysis Task Force Report-Part I. Value Health 2009;12:1044-52.

3. Hahn OM, Schilsky RL. Randomized controlled trials and comparative effectiveness research. J Clin Oncol 2012;30:4194-201.

4. Meyerhardt JA, Li L, Sanoff HK, et al. Effectiveness of bevacizumab with first-line combination chemotherapy for Medicare patients with stage IV colorectal cancer. J Clin Oncol 2012;30:608-15.

5. Prasad V, Kim C, Burotto M, et al. The strength of association between surrogate end points and survival in oncology: a systematic review of trial-level meta-analyses. JAMA Intern Med 2015;175:1389-98.

6. Visvanathan K, Levit LA, Raghavan D, et al. Untapped potential of observational research to inform clinical decision making: American society of clinical oncology research statement. J Clin Oncol 2017;35:1845-54.

7. Berger ML, Dreyer N, Anderson F, et al. Prospective observational studies to assess comparative effectiveness: the ISPOR good research practices task force report. Value Health 2012;15:217-30.

8. Morton SC, Costlow MR, Graff JS, et al. Standards and guidelines for observational studies: quality is in the eye of the beholder. J Clin Epidemiol 2016;71:3-10.

9. Rosenbaum PR, Rubin DB. Constructing a control group using multivariate matched sampling methods that incorporate the propensity score. The American Statistician 1985;39:33-8.

10. Stukel TA, Fisher ES, Wennberg DE, et al. Analysis of observational studies in the presence of treatment selection bias: effects of invasive cardiac management on AMI survival using propensity score and instrumental variable methods. JAMA 2007;297:278-85.

11. D'Agostino RB. Propensity score methods for bias reduction in the comparison of a treatment to a non-randomized control group. Stat Med 1998;17:2265-81.

12. Newhouse JP, McClellan M. Econometrics in outcomes research: the use of instrumental variables. Annu Rev Public Health 1998;19:17-34.

13. Institut National du Cancer (INCa). Information sur le cancer. http:// www.e-cancer.fr/ (Accessed 04 Jul 2017).

14. Early Breast Cancer Trialists' Collaborative Group (EBCTCG). Effects of chemotherapy and hormonal therapy for early breast cancer on recurrence and 15-year survival: an overview of the randomised trials. Lancet 2005;365:1687-717.

15. Binder-Foucard F, Belot A, Delafosse P, et al. Estimation nationale de l'incidence et de la mortalité par cancer en France entre 1980 et 2012. Partie 1 - Tumeurs solides. Saint-Maurice (Fra): Institut de veille sanitaire, 2013.

16. Delaloge S, Pérol D, Courtinard C, et al. Paclitaxel plus bevacizumab or paclitaxel as first-line treatment for HER2-negative metastatic breast cancer in a multicenter national observational study. Ann Oncol 2016;27:1725-32.

17. Gobbini E, Ezzalfani M, Dieras V, et al. Time trends of overall survival among metastatic breast cancer patients in the real-life ESME cohort. Eur J Cancer 2018;96:17-24.

18. Jacquet E, Lardy-Cléaud A, Pistilli B, et al. Endocrine therapy or chemotherapy as first-line therapy in hormone receptor-positive HER2-negative metastatic breast cancer patients. Eur J Cancer 2018;95:93-101.

19. Association des Epidémiologistes de Langue Française (ADELF). Déontologie et bonnes pratiques en épidémiologie, recommendations révisées. 2007.

20. ISPE. Guidelines for good pharmacoepidemiology practices (GPP). Pharmacoepidemiol Drug Saf 2008;17:200-8.

21. Largillier R, Ferrero JM, Doyen J, et al. Prognostic factors in 1,038 women with metastatic breast cancer. Ann Oncol 2008;19:2012-9.

22. Asselain B, De Rycke $Y$, Savignon A, et al. Parametric modelling to predict survival time to first recurrence for breast cancer. Phys Med Biol 2003;48:L31-3.

23. Broglio KR, Berry DA. Detecting an overall survival benefit that is derived from progression-free survival. J Natl Cancer Inst 2009;101:1642-9.

24. Pérol D, Robain M, Delaloge S, et al. Routinely collected data may usefully supplement randomised controlled data on treatment effects for mortality. BMJ 2016;355:i6745.

25. Berdaï D, Thomas-Delecourt F, Szwarcensztein K, et al. Requests for post-registration studies (PRS), patients follow-up in actual practice: Changes in the role of databases. Therapie 2018;73:13-24. 